\title{
Superior Orbital Fissure Syndrome: A Case Report
}

\author{
Heath H. Evans, D.D.S. ${ }^{1}$ Bradley A. Wurth, D.M.D. ${ }^{1}$ Kevin J. Penna, D.D.S. ${ }^{1}$ \\ ${ }^{1}$ Oral and Maxillofacial Surgery, Nassau University Medical Center, East \\ Meadow, New York \\ Craniomaxillofac Trauma Reconstruction 2012;5:115-120 \\ Address for correspondence and reprint requests Heath $\mathrm{H}$. Evans, \\ D.D.S., Oral and Maxillofacial Surgery, Nassau University Medical \\ Center, 2201 Hempstead Turnpike, Box 72, East Meadow, New York \\ 11554 (e-mail: hevans@numc.edu).
}
Abstract
Keywords
- trauma
- midface
- superior orbital fissure syndrome
- zygomaticomaxillary complex fracture

Superior orbital fissure syndrome is an infrequently encountered entity with a unique presentation and significant morbidity. This article reviews the background of the syndrome, treatments in the literature, and discusses a recent case with treatment strategy.
Superior orbital fissure syndrome (SOFS) is an interesting symptom complex, which poses difficult questions for the treating physicians. SOFS can arise from multiple etiologies and mechanisms. Presented in the following is a review of a recent traumatic incident confounded by SOFS.

\section{Case Presentation}

A 44-year-old man, status-post fall down eight concrete steps, was transported to the Nassau University Medical Center Emergency Department by emergency medical services and presented with a Glasgow Coma Scale of 13. Advanced Trauma Life Support protocol was followed. Neurosurgery service was consulted for a subdural hematoma, the Oral and Maxillofacial Surgery service was consulted to evaluate and treat multiple facial fractures and lacerations, and the Ophthalmology service was consulted to assess any visual disturbances resulting from the periorbital injuries. The patient was admitted to our institution on the Trauma service. The patient denied any medical or surgical history. He also reported taking no medications and having no known drug allergies or sensitivities. His social history was pertinent for alcohol consumption, but the patient denied tobacco or illicit drug use.

On initial head and neck physical examination, the patient displayed left periorbital edema and ecchymosis, left lid ptosis, limited mandibular range of motion, a palpable step at the left infraorbital rim, and decreased left facial projection (-Fig. 1). The ophthalmologic examination revealed no acute deficit in visual acuity, minimally elevated left intraocular pressure (left: 21 vs. right: 17 ) and anisocoria with the left pupil dilatated to $5 \mathrm{~mm}$ versus the right at $3 \mathrm{~mm}$. Also noted was an intact afferent pupillary response, paresthesia of the left frontal region, full restriction of motion of the left globe in all fields of gaze, and forced duction test was negative for entrapment of the extraocular muscles (-Fig. 2). Further ophthalmologic evaluation demonstrated no evidence of optic nerve edema, neuropathy, or retinal detachment.

Imaging included a maxillofacial computed tomography (CT) which revealed comminuted fractures of the anterior, posterolateral, and posteromedial walls of the left maxillary sinus with air fluid levels and a hematoma. In addition, fractures of the left orbital roof and lateral and inferior walls were noted. The globes were found to be intact, with no definitive evidence of muscle entrapment. However, disruption of the left superior orbital fissure was found (-Figs. 3 and 4 ).

Subsequently, the diagnosis was made of a left zygomaticomaxillary complex (ZMC) fracture with associated SOFS caused by compression of the fissure by bony segments. After discussion of treatment options, an open reduction with received

December 11, 2011

accepted after revision

January 17, 2012

published online

May 10, 2012
Copyright $\odot 2012$ by Thieme Medical Publishers, Inc., 333 Seventh Avenue, New York, NY 10001, USA. Tel: +1(212) 584-4662.
DOI http://dx.doi.org/ 10.1055/s-0032-1313363. ISSN 1943-3875. 


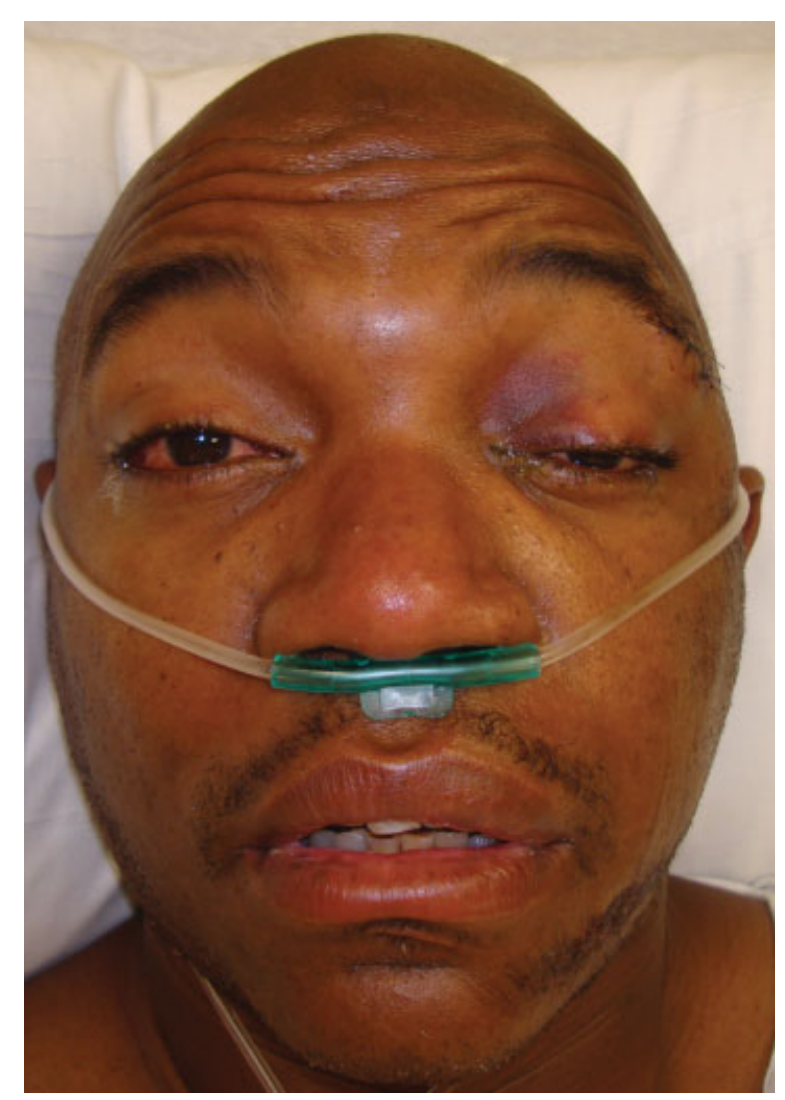

Figure 1 Initial presentation of the patient s/p fall. Note the typical presentation of the superior orbital fissure syndrome.

internal fixation of the left ZMC fracture was performed under general anesthesia using lateral brow and a maxillary vestibular incision with hopes of minimizing the bony impingement of the superior orbital fissure. The patient was given a perioperative dexamethasone taper beginning with an immediate preoperative dose of $10 \mathrm{mg}$. A postoperative maxillofacial CT was obtained to evaluate the reduction and reassess the left superior orbital fissure ( - Figs. 5 and $\mathbf{6}$ ). The patient was followed weekly by the Oral and Maxillofacial Surgery service as an outpatient. The patient's SOFS resolved completely (-Fig. 7).

\section{Discussion}

SOFS is an infrequently described and reported symptom complex. According to Kurzer and Patel, the syndrome was first described by Hirscfield in $1858 .^{1}$ SOFS consists of the following signs: ptosis of the upper eyelid, proptosis of the globe, ophthalmoplegia, fixation and dilatation of the pupil, and anesthesia of the upper eyelid and forehead. ${ }^{2}$

The superior orbital fissure serves as a pathway that allows communication between the orbit and the middle cranial fossa. ${ }^{3}$ It lies at the apex of the orbit, bounded medially by the lesser wing of the sphenoid, inferiorly and laterally by the greater wing of the sphenoid, and superiorly by the frontal bone. ${ }^{3}$ The fissure is reported to be $3 \times 22 \mathrm{~mm}^{4}$ and transmits the oculomotor, trochlear, and abducens nerves (cranial nerves III, IV, and VI), as well as the first three branches of the

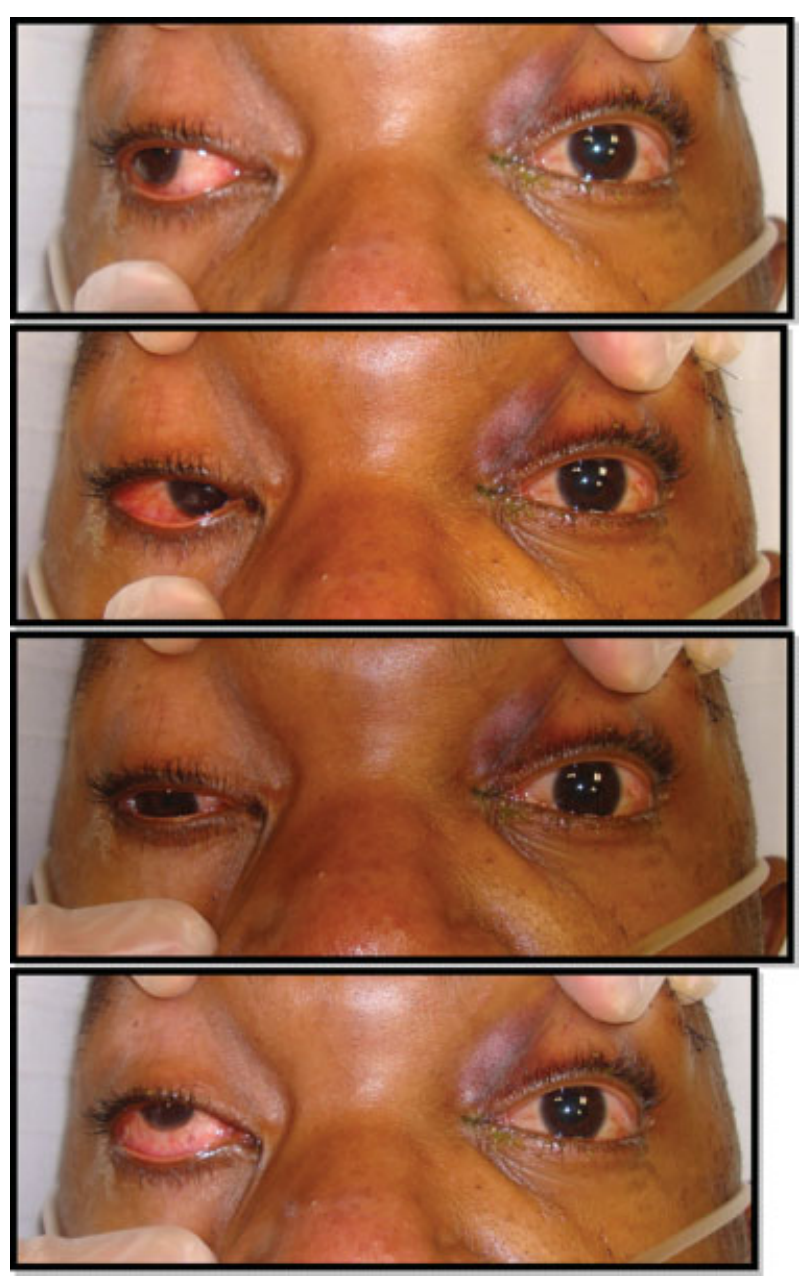

Figure 2 Clinical examination photos documenting ophthalmoplegia of the left eye.

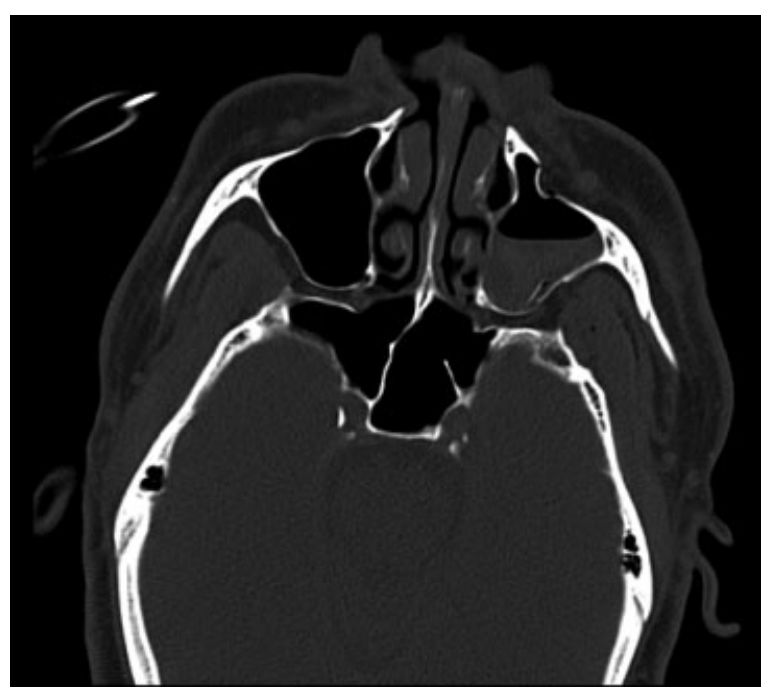

Figure 3 Maxillofacial computed tomography scan:, axial cut, bony window, at the level of the zygomatic arches showing left displaced zygomaticomaxillary complex fracture. 


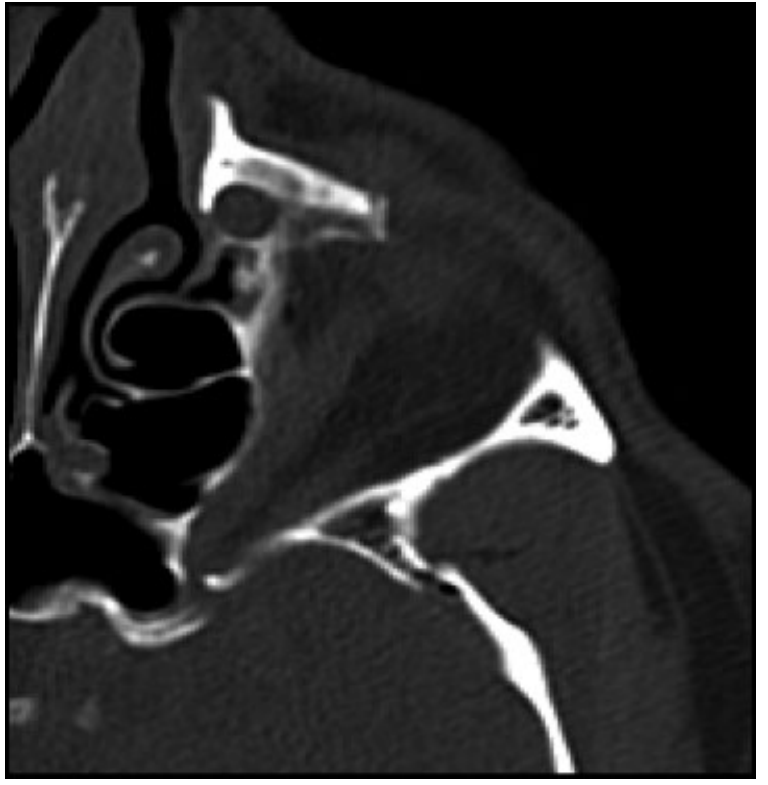

Figure 4 Enhanced view of the left superior orbital fissure from the previous image. Note the constriction and impingement of the superior orbital fissure.

trigeminal nerve: the frontal, lacrimal, and nasociliary nerves. $^{3,8}$ Also contained in the fissure is the inferior and superior ophthalmic veins as well as sympathetic filaments from the cavernous plexus. ${ }^{3}$ (-Fig. 8).

Numerous etiologies of the syndrome have been reported in the literature including syphilis, craniofacial fractures, hematoma of the cavernous sinus or retrobulbar space, infection, neoplasm, aneurysm of the internal carotid artery or arteriovenous fistulae, or idiopathic etiologies. ${ }^{1-8}$ Regardless of the etiology, the clinical symptoms are primarily the result of inflammation and compression of adjacent nervous

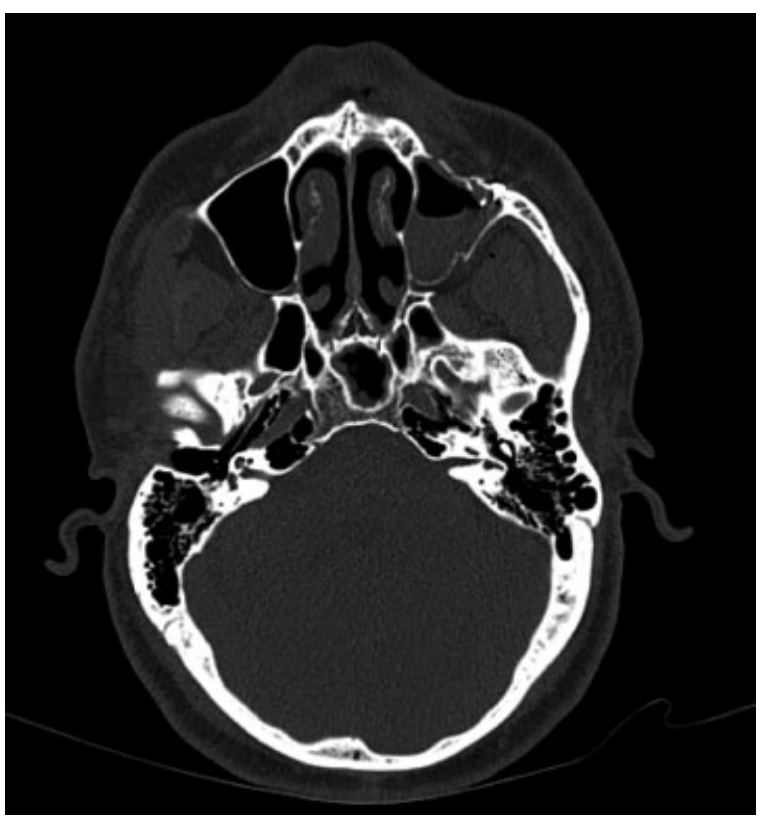

Figure 5 Postoperative maxillofacial computed tomography showing reduction of the left zygomaticomaxillary complex fractures.

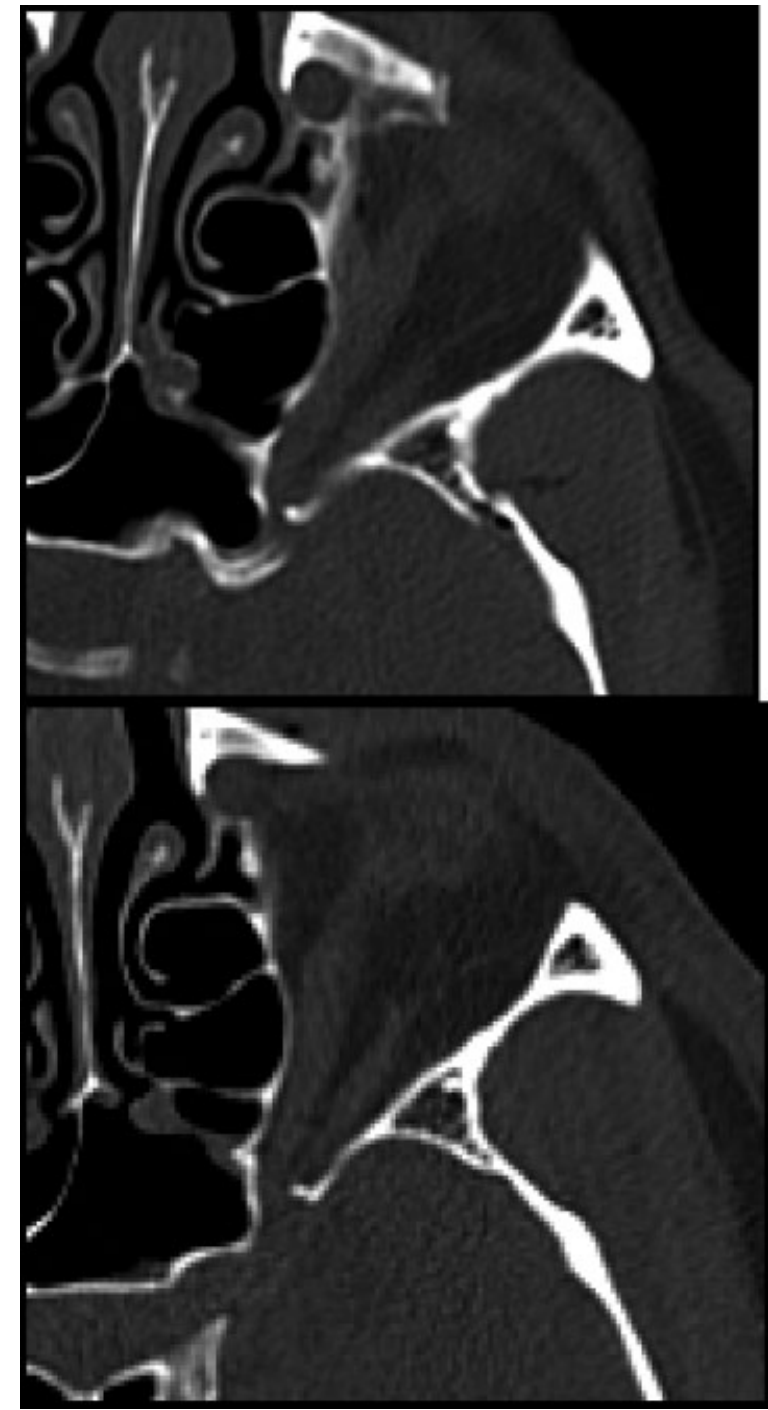

Figure 6 Comparison of the pre- and postoperative maxillofacial computed tomographic scans. Both images are axial cuts, bony windows, at the approximate level of the superior orbital fissure. Note the second image demonstrating widening of the superior orbital fissure, verified by radiological report, with decreased bony compression and excellent reduction of the fracture segment.

tissue. ${ }^{5}$ Lid ptosis is caused by either the involvement of the sympathetic fibers arising from the cavernous sinus, resulting in loss of tone of Mueller muscles, or the involvement of the somatic efferent fibers that course along the superior branch of the oculomotor nerve, resulting in loss of tone of the levator palpebrae superioris muscle. ${ }^{3,5}$ The ophthalmoplegia is secondary to impairment of cranial nerves III, IV, and VI. ${ }^{1-8}$ Disturbance of the lacrimal and frontal nerves leads to anesthesia of the forehead and upper eyelid. ${ }^{3,5}$ Compromise of the parasympathetic innervation, which travels with the oculomotor nerve, results in paralysis of the pupillary ciliary muscle. This paralysis causes dilatation, fixation, and loss of accommodation of the ipsilateral pupil. ${ }^{3,5,6}$ The proptosis can be attributed to the loss of tone of the extraocular muscles which normally exert a retracting force on the globe. ${ }^{3,5} \mathrm{~A}$ loss or disruption of visual acuity signals involvement of the optic 


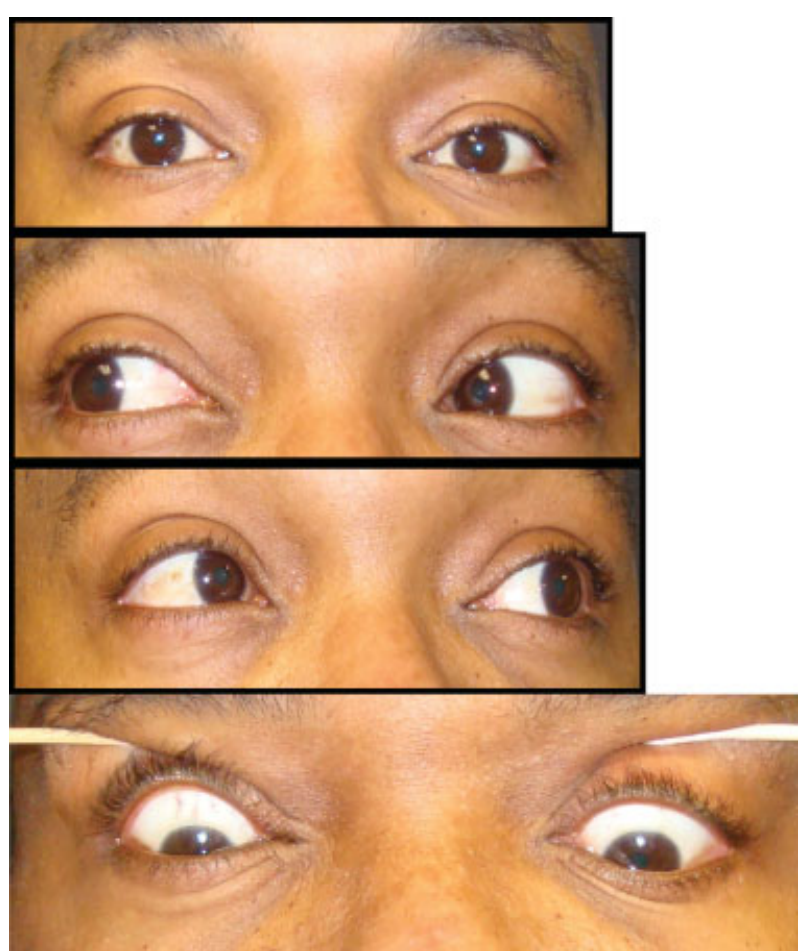

Figure 7 Postoperative clinical photos. Note the resolved periorbital edema and ecchymosis, complete resolution of anisocoria, and normal ocular movement.

nerve and when concomitant with SOFS is representative of orbital apex syndrome, first described by Kjoer, as reported by Kurzer and Patel. ${ }^{1}$

Treatment of SOFS remains poorly defined. Most authors agree that exploration is warranted in cases of neoplasm, physical impingement, infection, or retrobulbar hemorrhage, ${ }^{2,5-7}$ however, traumatic etiologies remain troublesome for the clinician. Although relatively few cases of SOFS exist in the literature, one author cited an incidence of 1 in 130 cases of LeFort II-III fractures ${ }^{6}$ at his institution. The occurrence of optic neuropathy is estimated between 0.5 and $5 \%$ of closed head traumas. ${ }^{9}$ Another author cited a SOFS incidence of $0.3 \%$ over a 14 -year period. ${ }^{8}$ Based on the

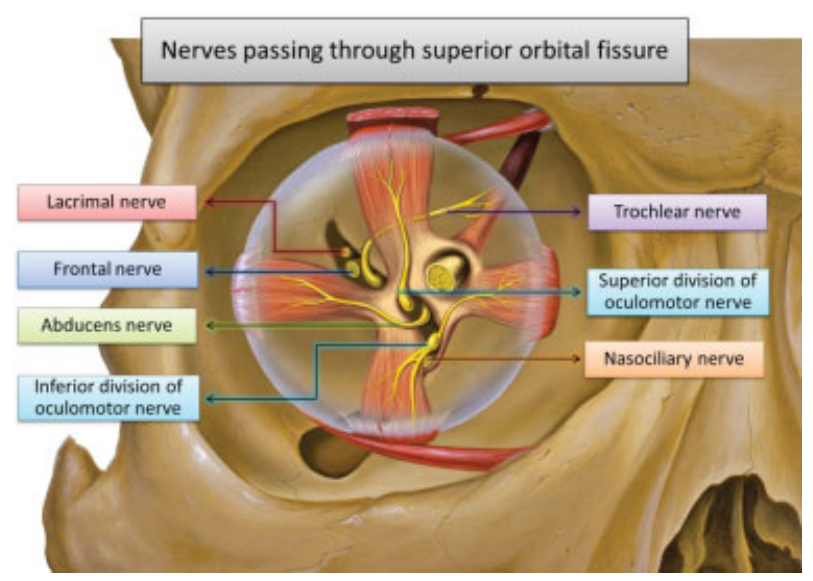

Figure 8 Anatomy of the superior orbital fissure and its contents. literature, open reduction and internal fixation of associated facial fractures may be beneficial and result in improvement of symptoms. ${ }^{3}$ If the SOFS is secondary to facial trauma, a complete or partial recovery can be expected without any intervention aimed at the fissure itself ${ }^{1,3,6}$ as long as the nerves are intact. The prognosis is obviously poor if the nerves have been severed or severely damaged by fractured bones. ${ }^{1}$ Also, varying doses of systemic corticosteroids have been advocated as treatment alone, or in conjunction with other modalities such as facial fracture reduction. ${ }^{3,4,7,8}$ One institution uses dexamethasone with a loading dose of $1 \mathrm{mg} /$ $\mathrm{kg}$ followed by $0.5 \mathrm{mg} / \mathrm{kg}$ that is tapered postoperatively ${ }^{3}$ after reduction of the fractures. Some authors advocate megadose steroid therapy on the order recommended by the 2nd National Acute Spinal Cord Injury Study. ${ }^{2}$ This involves dosing methylprednisolone $30 \mathrm{mg} / \mathrm{kg}$ followed by $5.4 \mathrm{mg} / \mathrm{kg} / \mathrm{h} .{ }^{10}$ The benefits of steroids appear to be from the antioxidant mechanism and/or the ability of such high doses to reduce edema and subsequent ischemia at the affected sites. ${ }^{11}$ Regardless of treatment, SOFS seems to improve or resolve within 6 months, with a plateau of nerve function recovery occurring at this time, ${ }^{2-8}$ unless there is a physical obstruction impeding recovery. There is, however, one case in the literature reviewed that documents a SOFS developing after open reduction and internal fixation of facial fractures, although the patient appeared to be anatomically unique with a narrow fissure. ${ }^{4}$

A case of facial trauma, specifically a ZMC fracture, complicated by SOFS is presented. The patient was treated with routine open reduction with internal fixation of fractures via the standard approaches in our institution. No specific instrumentation directly aimed at the superior orbital fissure was performed. Although we do not know whether the patient in this case would have experienced symptom resolution without surgical intervention as the literature suggests and discussed previously, ${ }^{1-3}$ it is clear that routine surgical treatment of ZMC fractures can have a positive effect on the superior orbital fissure if narrowed due to trauma and may at least hasten the resolution of SOFS in selected cases. In addition, the presence of SOFS in the setting of acute orbitofacial fractures, likely due to impingement of the structures of the superior orbital fissure by bony fragments or increasing edema, one should consider perioperative dexamethasone (not necessarily mega dose) administration and routine open reduction with internal fixation of the associated fractures in an attempt to reestablish proper dimensions of the superior orbital fissure. This case illustrates one treatment option for such cases and may provide useful information for clinicians and patients alike regarding outcomes.

\section{References}

1 Kurzer A, Patel MP. Superior orbital fissure syndrome associated with fractures of the zygoma and orbit. Plast Reconstr Surg 1979;64(5):715-719

2 Acartürk S, Seküçoğlu T, Kesiktäs E. Mega dose corticosteroid treatment for traumatic superior orbital fissure and orbital apex syndromes. Ann Plast Surg 2004;53(1):60-64 
3 Rohrich RJ, Hackney FL, Parikh RS. Superior orbital fissure syndrome: current management concepts. J Craniomaxillofac Trauma 1995;1(2):44-48

4 Fujiwara T, Matsuda K, Kubo T, Tomita K, Yano K, Hosokawa K. Superior orbital fissure syndrome after repair of maxillary and naso-orbito-ethmoid fractures: a case study. J Plast Reconstr Aesthet Surg 2009;62(12):e565-e569

5 Hedstrom J, Parsons J, Maloney PL, Doku HC. Superior orbital fissure syndrome: report of case. J Oral Surg 1974;32(3):198-201

6 Zachariades N, Vairaktaris E, Papavassiliou D, Papademetriou I, Mezitis M, Triantafyllou D. The superior orbital fissure syndrome.J Maxillofac Surg 1985;13(3):125-128

7 Campiglio GL, Signorini M, Candiani P. Superior orbital fissure syndrome complicating zygomatic fractures. Pathogenesis and report of a case. Case report. Scand J Plast Reconstr Surg Hand Surg 1995;29(1):69-72

8 Chen CT, Wang TY, Tsay PK, Huang F, Lai JP, Chen YR. Traumatic superior orbital fissure syndrome: assessment of cranial nerve recovery in 33 cases. Plast Reconstr Surg 2010;126(1):205-212

9 Steinsapir KD, Goldberg RA. Traumatic optic neuropathy. Surv Ophthalmol 1994;38(6):487-518

10 Bracken MB, Shepard MJ, Collins WF, et al. A randomized, controlled trial of methylprednisolone or naloxone in the treatment of acute spinal-cord injury. Results of the Second National Acute Spinal Cord Injury Study. N Engl J Med 1990;322(20):1405-1411

11 Hall ED, Braughler JM. Glucocorticoid mechanisms in acute spinal cord injury: a review and therapeutic rationale. Surg Neurol 1982;18(5):320-327 